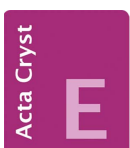

CRYSTALLOGRAPHIC COMMUNICATIONS

ISSN 2056-9890

Received 21 December 2020

Accepted 17 June 2021

Edited by G. Diaz de Delgado, Universidad de Los Andes, Venezuela

Keywords: 2-picoline complex; inorganic supramolecular chemistry; crystal structure; hydrogen bonding.

CCDC reference: 2090586

Supporting information: this article has supporting information at journals.iucr.org/e

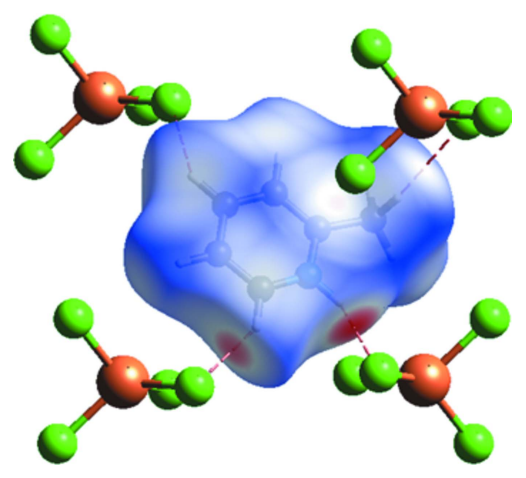

OPEN $\partial$ ACCESS

\section{Bis(2-methylpyridinium) tetrachloridocuprate(II): synthesis, structure and Hirshfeld surface analysis}

\author{
Tahir Mehmood, Rajesh S. Bhosale* and J. Prakasha Reddy* \\ Department of Chemistry, School of Sciences, Indrashil University, Rajpur, Gujarat, 382740, India. *Correspondence \\ e-mail: rajeshbhosale24@gmail.com, j.prakashareddy@gmail.com
}

The title compound, $\left(\mathrm{C}_{6} \mathrm{H}_{8} \mathrm{~N}\right)_{2}\left[\mathrm{CuCl}_{4}\right]$, crystallizes in the monoclinic space group $I 2 / c$. The coordination around the copper atom is a distorted tetrahedron. The 2-methylpyridinium ion $\left(\mathrm{C}_{6} \mathrm{H}_{8} \mathrm{~N}^{+}\right)$interacts with the tetrachlorocuprate anion through $\mathrm{N}-\mathrm{H} \cdots \mathrm{Cl}$ and $\mathrm{C}-\mathrm{H}$ (phenyl) $\cdots \mathrm{Cl}$ contacts, forming a hydrogen-bonded layer-like structure. The supramolecular structure is further stabilized by $\mathrm{C}-\mathrm{H}($ methyl $) \cdots \mathrm{Cl}$ interactions between the layers.

\section{Chemical context}

Supramolecular organic and inorganic chemistry have been studied both from the fundamental as well as the application point of view, which is evident from the literature (Ziach et al., 2018; Thorat et al., 2013; Burslem et al., 2016). With the surge in the number of compounds reported, potential applications of supramolecular inorganic materials in energy storage, separation, catalysis, sensors, molecular magnets, optoelectronic materials, etc., have attracted greater attention in recent years (Mueller et al., 2006; Wan et al., 2006; Férey et al., 2003; James, 2003; Eddaoudi et al., 2002; Ruben et al., 2005, Kitagawa et al., 2004, Stavila et al., 2014). Because of the divergent combination of ligands and metal salts, an enormous number of structural architectures with different sizes and shapes could be constructed (Moulton \& Zaworotko, 2001). The special characteristics and features such as ease of synthesis of the material, geometrically well-defined structures, exceptional tunability, post-synthetic modification, along with robustness of the material resulting from strong directional bonding, produce new opportunities and offer a unique platform amenable to the synthesis of more and more functional solids. For example, Adams et al. (2005) reported the synthesis of coordination compounds using a new synthetic route involving a thermal dehydrochlorination reaction in crystals of a pyridinium chlorometallate bicomponent system, i.e., anionic metal complexes and organic cations.

As part of ongoing studies in our group (PrakashaReddy \& Pedireddi, 2007; Reddy et al., 2014), the synthesis of coordination complexes using pyridine ligands has been reported. Hence, we further extended our studies to utilize the pyridinium ligand and to study in situ the single-crystal-to-singlecrystal transition (SCSCT) to investigate the reaction mechanism. In our endeavours to synthesize new functional solids, using a transition-metal anion and a pyridinium cation, we have chosen the $\mathrm{CuCl}_{2}$ and 2-methylpyridinium salt complex. Herein, we report the synthesis and crystal structure 
of a bis(2-methylpyridinium) tetrachlorocuprate coordination complex.<smiles>[3H]C(Cl)(Cl)Cc1ccccn1</smiles>

\section{Structural commentary}

The title complex crystallizes in the monoclinic space group I2/c. Since the $\mathrm{Cu}^{2+}$ cation occupies a special position, the asymmetric unit consists of a 2-methylpyridinium cation, $[2-\mathrm{Me}(\mathrm{Py}) \mathrm{H}]^{+}$, and half of a tetrachlorocuprate(II) anion, $\left[\mathrm{CuCl}_{4}\right]^{2-}$. The molecular structure of the complex along with the atom-labelling scheme is shown in Fig. 1. Each copper center is four-coordinated by chlorine anions and adopts a distorted tetrahedral geometry. Structural analysis shows that the $\mathrm{Cl}-\mathrm{Cu}-\mathrm{Cl}$ angles vary from 98.55 (2) to 137.4 (3) ${ }^{\circ}$ with four angles smaller and two larger than the standard tetrahedral angle. A plausible reason for a larger deviation from the standard $109.5^{\circ}$ might be due to the $\mathrm{N}-\mathrm{H} \cdots \mathrm{Cl}$ and $\mathrm{C}-$ $\mathrm{H} \cdots \mathrm{Cl}$ interactions. A similar marked deviation from the standard tetrahedral angle has been previously observed by other research groups (Wyrzykowski et al., 2011; Jasrotia et al., 2018). The $\mathrm{Cu}-\mathrm{Cl}$ bond lengths $[\mathrm{Cu} 1-\mathrm{Cl} 1=2.250$ (1) $\AA$, $\mathrm{Cu} 1-\mathrm{Cl} 2=2.249$ (1) $\AA$ ] agree well with those reported for other structures (Marsh et al., 1982; Dodds et al., 2018; Molano et al., 2020; Reddy, 2020). The intramolecular $\mathrm{C}_{\mathrm{ar}}-\mathrm{C}_{\mathrm{ar}}$ bond lengths in the $[2-\mathrm{Me}(\mathrm{Py}) \mathrm{H}]^{+}$fall in the range $1.370(3)-$ 1.395 (3) $\AA$. The $\mathrm{N} 1-\mathrm{C} 2$ and $\mathrm{N} 1-\mathrm{C} 6$ bond lengths are 1.350 (2) and 1.346 (2) A, respectively.
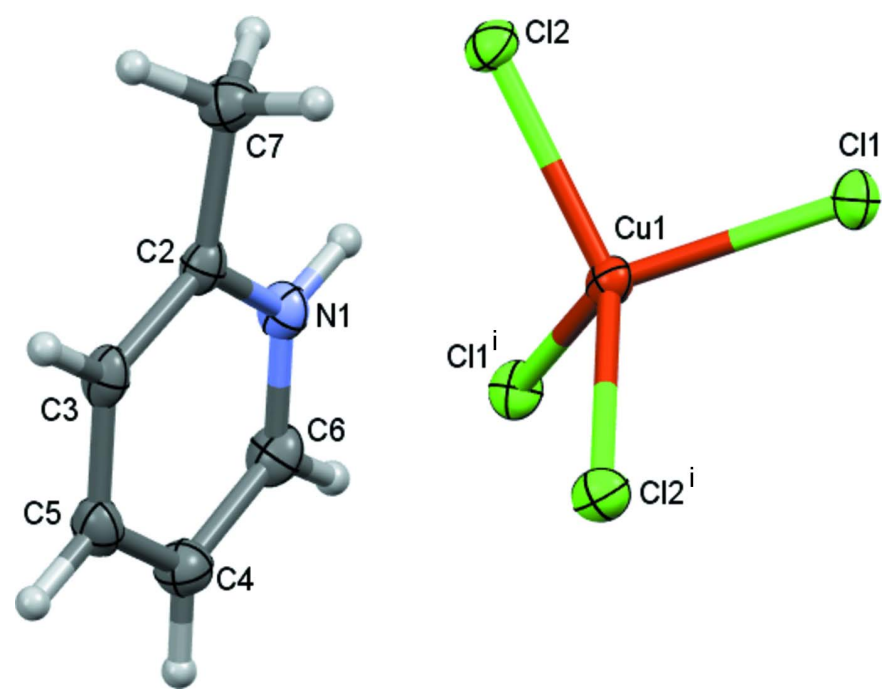

Figure 1

The molecular structure of the title compound, showing the atom labelling and displacement ellipsoids drawn at the $50 \%$ probability level. Hydrogen atoms are shown as small spheres of arbitrary size. [Symmetry code: (i) $1-x, y, \frac{1}{2}-z$.]
Table 1

Hydrogen-bond geometry $\left(\AA{ }^{\circ}\right)$.

\begin{tabular}{|c|c|c|c|c|}
\hline$D-\mathrm{H} \cdots A$ & $D-\mathrm{H}$ & $\mathrm{H} \cdots A$ & $D \cdots A$ & $D-\mathrm{H} \cdots A$ \\
\hline $\mathrm{N} 1-\mathrm{H} 1 \cdots \mathrm{Cl} 1^{\mathrm{i}}$ & 0.88 & 2.93 & $3.4297(16)$ & 118 \\
\hline $\mathrm{N} 1-\mathrm{H} 1 \cdots \mathrm{Cl} 2^{\mathrm{i}}$ & 0.88 & 2.41 & $3.2050(16)$ & 150 \\
\hline $\mathrm{C} 6-\mathrm{H} 6 \cdots \mathrm{Cl}^{\mathrm{ii}}$ & 0.95 & 2.62 & $3.453(2)$ & 147 \\
\hline $\mathrm{C} 7-\mathrm{H} 7 A \cdots \mathrm{Cl} 2$ & 0.98 & 2.92 & $3.850(2)$ & 159 \\
\hline $\mathrm{C} 7-\mathrm{H} 7 B \cdots \mathrm{Cl} 2^{\mathrm{iii}}$ & 0.98 & 2.98 & $3.872(2)$ & 151 \\
\hline
\end{tabular}

\section{Supramolecular features and Hirshfeld surface analysis}

In the crystal, complex molecules related by the twofold rotation axis are connected by pairs of $\mathrm{N}-\mathrm{H} \cdots \mathrm{Cl}$ and $\mathrm{C}_{\mathrm{ar}}-$ $\mathrm{H} \cdots \mathrm{Cl}$ interactions through a protonated $\mathrm{N}$ and an aromatic hydrogen attached to the carbon atom with the chloride ligand bonded to copper, forming a monomeric unit. These units interact with adjacent ones through $\mathrm{C}_{\mathrm{ar}}-\mathrm{H} \cdots \mathrm{Cl}$ hydrogen bonding (Table 1, Fig. 2). The $\mathrm{N}-\mathrm{H} \cdots \mathrm{Cl}$ and $\mathrm{C}-\mathrm{H} \cdots \mathrm{Cl}$ distances and associated bond angles lie within the ranges observed for other similar interactions reported in the literature (Adams et al., 2005; Vittaya et al., 2015; Wyrzykowski et

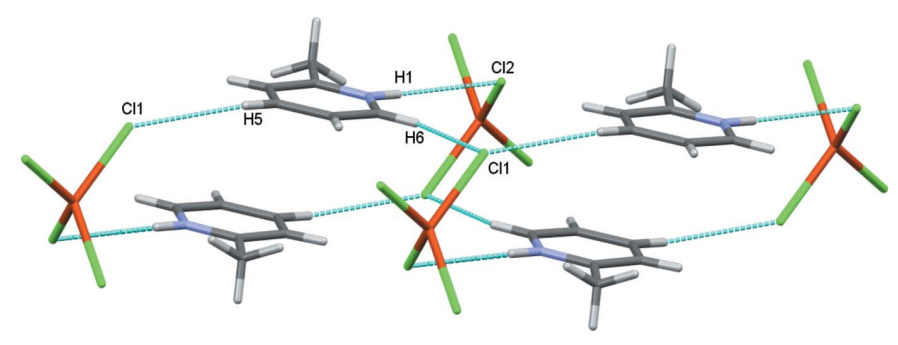

Figure 2

The $\mathrm{N}-\mathrm{H} \cdots \mathrm{Cl}$ and $\mathrm{C}-\mathrm{H} \cdots \mathrm{Cl}$ interactions between cations and anions in the crystal structure of the title compound.

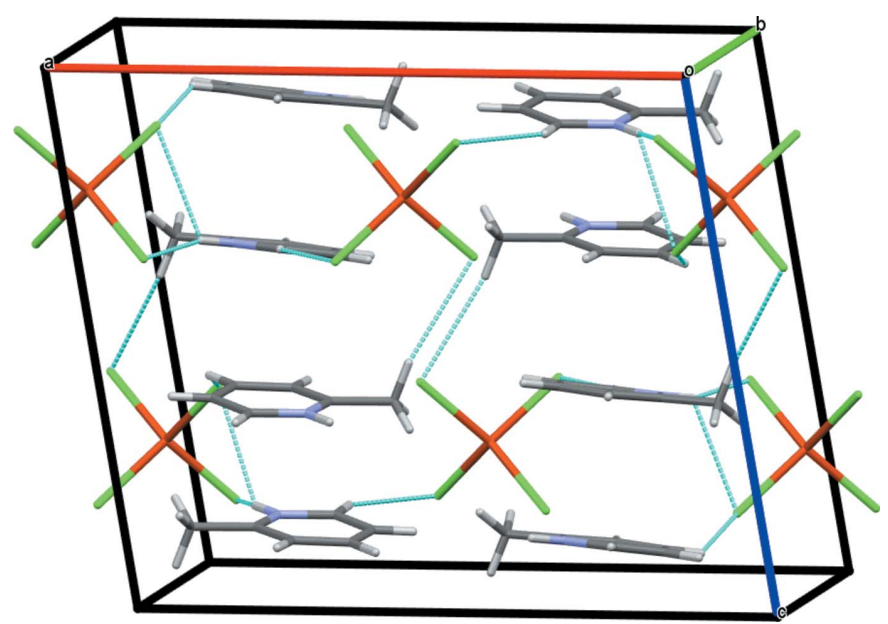

Figure 3

The crystal packing of the title compound viewed along the $b$ axis with intermolecular contacts shown as dashed lines. 


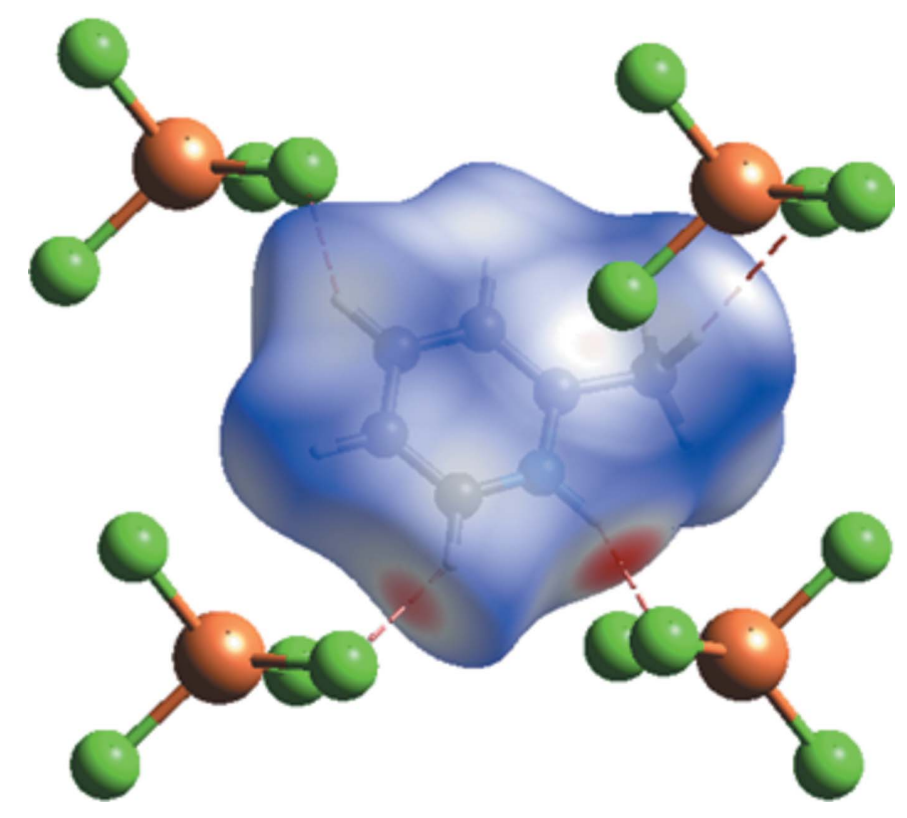

Figure 4

Hirshfeld surface mapped over $d_{\text {norm }}$ highlighting the regions of $\mathrm{N}-$ $\mathrm{H} \cdots \mathrm{Cl}$ and $\mathrm{C}-\mathrm{H} \cdots \mathrm{Cl}$ intermolecular contacts.

al., 2011; Jasrotia et al., 2018). The supramolecular structure is further stabilized by $\mathrm{C}_{\text {methyl }}-\mathrm{H} \cdots \mathrm{Cl}$ interactions involving hydrogens of the methyl group and chlorides bonded to copper, generating layers along the crystallographic $b$ axis (Fig. 3).

To further investigate the intermolecular interactions present in the title compound, a Hirshfeld surface analysis was performed and the two-dimensional fingerprint plots were generated with Crystal Explorer17 (Turner et al., 2017). The Hirshfeld surface mapped over $d_{\text {norm }}$ and corresponding colours representing various interactions are shown in Fig. 4. The red points on the surface correspond to the $\mathrm{N}-\mathrm{H} \cdots \mathrm{Cl}$ and $\mathrm{C}-\mathrm{H} \cdots \mathrm{Cl}$ interactions. The two-dimensional fingerprint plots (McKinnon et al., 2007) are shown in Fig. 5. On the Hirshfeld surface, the largest contribution (53.1\%) comes from the weak van der Waals $\mathrm{H} \cdots \mathrm{H}$ contacts. The interaction of $d_{\text {norm }}$ on the two-dimensional fingerprint plot shows two spikes; each one corresponds to $\mathrm{H} \cdot \mathrm{H}(39 \%)$ and $\mathrm{H} \cdot \mathrm{Cl} /$ $\mathrm{Cl} \cdots \mathrm{H}(32.5 \%)$ respectively. The $\mathrm{H} \cdots \mathrm{Cl}$ interaction highlights the hydrogen bond between adjacent moieties in the crystal structure. The $\mathrm{C} \cdots \mathrm{H} / \mathrm{H} \cdots \mathrm{C}(16.5 \%)$ interactions appear as two shoulders. These interactions play a crucial role in the overall stabilization of the crystal packing.

\section{Database survey}

A search of the Cambridge Structural Database (CSD, Version 5.41, update of August 2020; Groom et al., 2016) revealed two related complexes containing 2-methylpyridinium: [2-methylpyridinium tetrachloroferrate(III)] (CCDC refcode WAYJEN; Wyrzykowski et al., 2011) and [bis(2-methyl-pyridinium) tetrachloro-zinc(II)] (CCDC refcode WIPCUW; Jasrotia et al., 2018). The molecular structures of both WAYJEN and WIPCUW display threedimensional supramolecular networks arising from $\mathrm{N}-\mathrm{H} \cdots \mathrm{Cl}$ and $\mathrm{C}-\mathrm{H} \cdots \mathrm{Cl}$ interactions. In addition, the search also revealed a 2-methylpyridine and copper chloride complex: [dichloro-bis(2-methylpyridine)Cu(II)] (CCDC refcode CMPYCU01; Marsh et al., 1982) and [aqua-dichloro-bis(2methylpyridine) $\mathrm{Cu}(\mathrm{II})$ ] (CCDC refcode BIJWUM; Marsh et al., 1982) and a very recently published dichloridomethanolbis(2-methylpyridine)Cu(II) complex (Reddy, 2020). All of these structures display three-dimensional supramolecular networks stabilized by $\mathrm{C}-\mathrm{H} \cdots \mathrm{Cl}$ and $\mathrm{O}-\mathrm{H} \cdots \mathrm{Cl}$ interactions.

\section{Synthesis and crystallization}

Both 2-methylpyridine and anhydrous copper(II) chloride obtained from Aldrich were used for the reaction. Anhydrous copper(II) chloride $(0.495 \mathrm{~g}, 0.005 \mathrm{~mol})$ was dissolved in $10 \mathrm{ml}$ of distilled water. To this solution, 2-methylpyridine $(0.93 \mathrm{~g}$, $0.01 \mathrm{~mol}$ ) was added followed by addition of few drops of $\mathrm{HCl}$ (36\%) and the resulting mixture was stirred for $\sim 30 \mathrm{~min}$. at room temperature. The solution was then allowed to stand at room temperature for a few hours before being filtered and left at room temperature for crystallization. Block-shaped, pale-yellow-coloured crystals were obtained after $36 \mathrm{~h}$.

\section{Refinement}

Crystal data, data collection and structure refinement details are summarized in Table 2. $\mathrm{H}$ atoms were placed in calculated
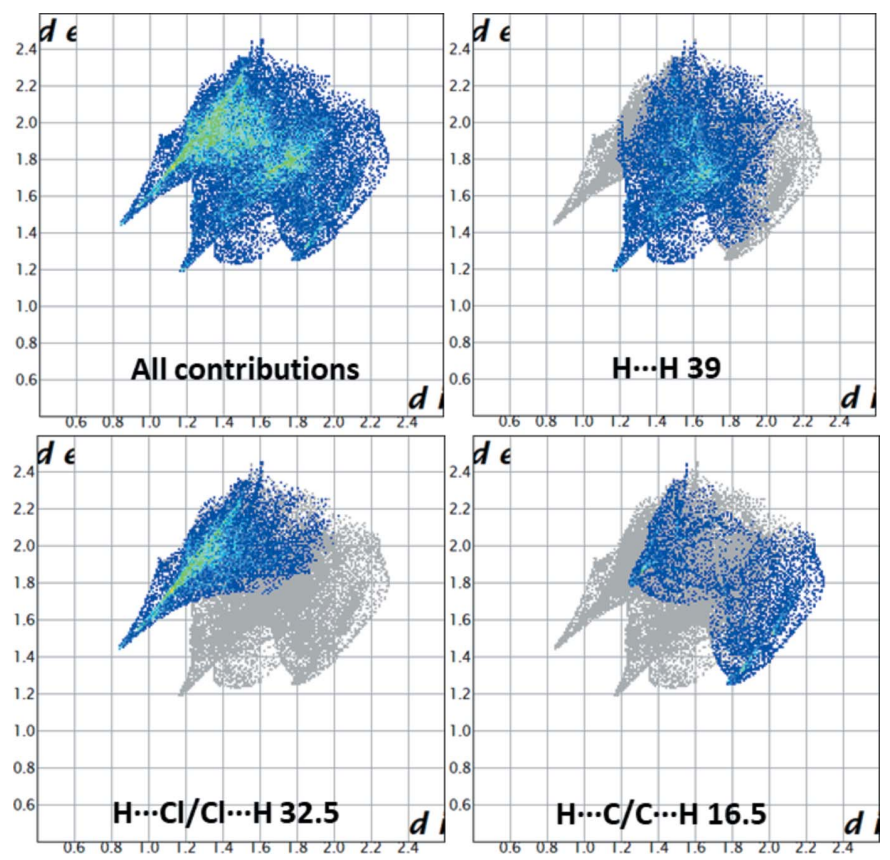

Figure 5

The full two-dimensional fingerprint plot for the organic cation in the title compound and those delineated into $\mathrm{H} \cdots \mathrm{H}(39 \%), \mathrm{Cl} \cdots \mathrm{H} / \mathrm{H} \cdots \mathrm{Cl}$ $(32.5 \%)$ and $\mathrm{C} \cdots \mathrm{H} / \mathrm{H} \cdots \mathrm{C}(16.5 \%)$ contacts. 
Table 2

Experimental details.

\begin{tabular}{|c|c|}
\hline \multicolumn{2}{|l|}{ Crystal data } \\
\hline Chemical formula & $\left(\mathrm{C}_{6} \mathrm{H}_{8} \mathrm{~N}\right)_{2}\left[\mathrm{CuCl}_{4}\right]$ \\
\hline$M_{\mathrm{r}}$ & 393.62 \\
\hline Crystal system, space group & Monoclinic, $I 2 / c$ \\
\hline Temperature $(\mathrm{K})$ & 120 \\
\hline$a, b, c(\AA)$ & $15.2354(8), 8.3683(3), 12.8372(6)$ \\
\hline$\beta\left(^{\circ}\right)$ & $99.205(5)$ \\
\hline$V\left(\AA^{3}\right)$ & $1615.60(13)$ \\
\hline$Z$ & 4 \\
\hline Radiation type & Мо $K \alpha$ \\
\hline$\mu\left(\mathrm{mm}^{-1}\right)$ & 2.00 \\
\hline Crystal size (mm) & $0.32 \times 0.27 \times 0.25$ \\
\hline \multicolumn{2}{|l|}{ Data collection } \\
\hline Diffractometer & Agilent Xcalibur, Sapphire3 \\
\hline Absorption correction & $\begin{array}{l}\text { Analytical (Crys Alis PRO; Agilent, } \\
\text { 2014) }\end{array}$ \\
\hline$T_{\min }, T_{\max }$ & $0.848,0.965$ \\
\hline $\begin{array}{l}\text { No. of measured, independent and } \\
\text { observed }[I>2 \sigma(I)] \text { reflections }\end{array}$ & $24556,2821,2324$ \\
\hline$R_{\text {int }}$ & 0.071 \\
\hline$(\sin \theta / \lambda)_{\max }\left(\AA^{-1}\right)$ & 0.758 \\
\hline \multicolumn{2}{|l|}{ Refinement } \\
\hline$R\left[F^{2}>2 \sigma\left(F^{2}\right)\right], w R\left(F^{2}\right), S$ & $0.036,0.081,1.08$ \\
\hline No. of reflections & 2821 \\
\hline No. of parameters & 88 \\
\hline $\mathrm{H}$-atom treatment & $\mathrm{H}$-atom parameters constrained \\
\hline$\Delta \rho_{\max }, \Delta \rho_{\min }\left(\mathrm{e} \AA^{-3}\right)$ & $0.58,-0.58$ \\
\hline
\end{tabular}

Computer programs: CrysAlis PRO (Agilent, 2014), SUPERFLIP (Palatinus \& Chapuis, 2007; Palatinus \& van der Lee, 2008; Palatinus et al., 2012), SHELXL (Sheldrick, 2015) and OLEX2 (Dolomanov et al., 2009).

positions with $\mathrm{C}-\mathrm{H}=0.93-0.96 \AA$ and $\mathrm{N}-\mathrm{H}=0.88 \AA$ and refined as riding with fixed isotropic displacement parameters $\left[U_{\text {iso }}(\mathrm{H})=1.2-1.5 U_{\text {eq }}(\mathrm{C}, \mathrm{N})\right]$.

\section{Acknowledgements}

We thank Dr J. S. Yadav, Provost and Director (R\&D) for his support and encouragement.

\section{Funding information}

Funding for this research was provided by: Indrashil University.

\section{References}

Adams, C. J., Crawford, P. C., Orpen, A. G., Podesta, T. J. \& Salt, B. (2005). Chem. Commun. pp. 2457-2458.

Agilent (2014). CrysAlis PRO. Agilent Technologies Ltd, Yarnton, England.

Burslem, G. M., Kyle, H. F., Prabhakaran, P., Breeze, A. L., Edwards, T. A., Warriner, S. L., Nelson, A. \& Wilson, A. J. (2016). Org. Biomol. Chem. 14, 3782-3786.
Dodds, C. A. \& Kennedy, A. R. (2018). Acta Cryst. E74, 1369-1372.

Dolomanov, O. V., Bourhis, L. J., Gildea, R. J., Howard, J. A. K. \& Puschmann, H. (2009). J. Appl. Cryst. 42, 339-341.

Eddaoudi, M., Kim, J., Rosi, N., Vodak, D., Wachter, J., O'Keeffe, M. \& Yaghi, O. M. (2002). Science, 295, 469-472.

Férey, G., Latroche, M., Serre, C., Millange, F., Loiseau, T. \& Percheron-Guégan, A. (2003). Chem. Commun. pp. 2976-2977.

Groom, C. R., Bruno, I. J., Lightfoot, M. P. \& Ward, S. C. (2016). Acta Cryst. B72, 171-179.

James, S. L. (2003). Chem. Soc. Rev. 32, 276-288.

Jasrotia, D., Verma, S. K., Sridhar, B., Alvi, P. A. \& Kumar, A. (2018). Mater. Chem. Phys. 207, 98-104.

Kitagawa, S., Kitaura, R. \& Noro, S. I. (2004). Angew. Chem. Int. Ed. 43, 2334-2375.

Marsh, W. E., Hatfield, W. E. \& Hodgson, D. J. (1982). Inorg. Chem. 21, 2679-2684.

McKinnon, J. J., Jayatilaka, D. \& Spackman, M. A. (2007). Chem. Commun. pp. 3814-3816.

Molano, M. F., Lorett Velasquez, V. P., Erben, M. F., Nossa González, D. L., Loaiza, A. E., Echeverría, G. A., Piro, O. E., Tobón, Y. A., Ben Tayeb, K. \& Gómez Castaño, J. A. (2020). Acta Cryst. E76, 148-154.

Moulton, B. \& Zaworotko, M. J. (2001). Chem. Rev. 101, 1629-1658.

Mueller, U., Schubert, M., Teich, F., Puetter, H., Schierle-Arndt, K. \& Pastré, J. (2006). J. Mater. Chem. 16, 626-636.

Palatinus, L. \& Chapuis, G. (2007). J. Appl. Cryst. 40, 786-790.

Palatinus, L., Prathapa, S. J. \& van Smaalen, S. (2012). J. Appl. Cryst. 45, 575-580.

Palatinus, L. \& van der Lee, A. (2008). J. Appl. Cryst. 41, 975-984.

PrakashaReddy, J. \& Pedireddi, V. R. (2007). Eur. J. Inorg. Chem. pp. $1150-1158$.

Reddy, J. P. (2020). Acta Cryst. E76, 1771-1774.

Reddy, J. P., Swain, D. \& Pedireddi, V. R. (2014). Cryst. Growth Des. 14, 5064-5071.

Ruben, M., Ziener, U., Lehn, J. M., Ksenofontov, V., Gütlich, P. \& Vaughan, G. B. M. (2005). Chem. Eur. J. 11, 94-100.

Sheldrick, G. M. (2015). Acta Cryst. C71, 3-8.

Stavila, V., Talin, A. A. \& Allendorf, M. D. (2014). Chem. Soc. Rev. 43, 5994-6010.

Thorat, V. H., Ingole, T. S., Vijayadas, K. N., Nair, R. V., Kale, S. S., Ramesh, V. V. E., Davis, H. C., Prabhakaran, P., Gonnade, R. G., Gawade, R. L., Puranik, V. G., Rajamohanan, P. R. \& Sanjayan, G. J. (2013). Eur. J. Org. Chem. pp. 3529-3542.

Turner, M. J., McKinnon, J. J., Wolff, S. K., Grimwood, D. J., Spackman, P. R., Jayatilaka, D. \& Spackman, M. A. (2017). CrystalExplorer17. The University of Western Australia.

Vittaya, L., Leesakul, N., Saithong, S. \& Chainok, K. (2015). Acta Cryst. E71, m201-m202.

Wan, Y., Yang, H. \& Zhao, D. (2006). Acc. Chem. Res. 39, 423-432.

Wyrzykowski, D., Wera, M., Sikorski, A., Jacewicz, D. \& Chmurzyński, L. (2011). Cent. Eur. J. Chem. 9, 1096-1101.

Ziach, K., Chollet, C., Parissi, V., Prabhakaran, P., Marchivie, M., Corvaglia, V., Bose, P. P., Laxmi-Reddy, K., Godde, F., Schmitter, J.-M., Chaignepain, S., Pourquier, P. \& Huc, I. (2018). Nat. Chem. 10, 511-518. 


\section{supporting information}

Acta Cryst. (2021). E77, 726-729 [https://doi.org/10.1107/S2056989021006277]

\section{Bis(2-methylpyridinium) tetrachloridocuprate(II): synthesis, structure and}

\section{Hirshfeld surface analysis}

\section{Tahir Mehmood, Rajesh S. Bhosale and J. Prakasha Reddy}

Computing details

Data collection: CrysAlis PRO (Agilent, 2014); cell refinement: CrysAlis PRO (Agilent, 2014); data reduction: CrysAlis PRO (Agilent, 2014); program(s) used to solve structure: Superflip (Palatinus \& Chapuis, 2007; Palatinus \& van der Lee, 2008; Palatinus et al., 2012); program(s) used to refine structure: SHELXL (Sheldrick, 2015); molecular graphics: OLEX2 (Dolomanov et al., 2009); software used to prepare material for publication: OLEX2 (Dolomanov et al., 2009).

Bis(2-methylpyridinium) tetrachloridocuprate(II)

\section{Crystal data}

$\left(\mathrm{C}_{6} \mathrm{H}_{8} \mathrm{~N}\right)_{2}\left[\mathrm{CuCl}_{4}\right]$

$M_{r}=393.62$

Monoclinic, $I 2 / \mathrm{c}$

$a=15.2354(8) \AA$

$b=8.3683(3) \AA$

$c=12.8372(6) \AA$

$\beta=99.205(5)^{\circ}$

$V=1615.60(13) \AA^{3}$

$Z=4$

\section{Data collection}

Agilent Xcalibur, Sapphire3 diffractometer

Detector resolution: 16.1511 pixels $\mathrm{mm}^{-1}$ $\omega$ scans

Absorption correction: analytical

(CrysAlisPro; Agilent, 2014)

$T_{\min }=0.848, T_{\max }=0.965$

24556 measured reflections

\section{Refinement}

Refinement on $F^{2}$

Least-squares matrix: full

$R\left[F^{2}>2 \sigma\left(F^{2}\right)\right]=0.036$

$w R\left(F^{2}\right)=0.081$

$S=1.08$

2821 reflections

88 parameters

0 restraints

Primary atom site location: iterative
$F(000)=796$

$D_{\mathrm{x}}=1.618 \mathrm{Mg} \mathrm{m}^{-3}$

Mo $K \alpha$ radiation, $\lambda=0.71073 \AA$

Cell parameters from 5462 reflections

$\theta=2.9-32.5^{\circ}$

$\mu=2.00 \mathrm{~mm}^{-1}$

$T=120 \mathrm{~K}$

Blocks, pale yellow

$0.32 \times 0.27 \times 0.25 \mathrm{~mm}$

2821 independent reflections

2324 reflections with $I>2 \sigma(I)$

$R_{\text {int }}=0.071$

$\theta_{\text {max }}=32.6^{\circ}, \theta_{\min }=2.9^{\circ}$

$h=-22 \rightarrow 22$

$k=-11 \rightarrow 12$

$l=-19 \rightarrow 19$

Hydrogen site location: inferred from neighbouring sites

$\mathrm{H}$-atom parameters constrained

$w=1 /\left[\sigma^{2}\left(F_{\mathrm{o}}^{2}\right)+(0.0308 P)^{2}+1.4147 P\right]$

where $P=\left(F_{\mathrm{o}}^{2}+2 F_{\mathrm{c}}^{2}\right) / 3$

$(\Delta / \sigma)_{\max }<0.001$

$\Delta \rho_{\max }=0.58 \mathrm{e} \AA^{-3}$

$\Delta \rho_{\min }=-0.58$ e $\AA^{-3}$ 


\section{Special details}

Geometry. All esds (except the esd in the dihedral angle between two 1.s. planes) are estimated using the full covariance matrix. The cell esds are taken into account individually in the estimation of esds in distances, angles and torsion angles; correlations between esds in cell parameters are only used when they are defined by crystal symmetry. An approximate (isotropic) treatment of cell esds is used for estimating esds involving l.s. planes.

Fractional atomic coordinates and isotropic or equivalent isotropic displacement parameters $\left(\AA^{2}\right)$

\begin{tabular}{lllll}
\hline & $x$ & $y$ & $z$ & $U_{\text {iso }} / U_{\text {eq }}$ \\
\hline Cu1 & 0.500000 & $0.22261(4)$ & 0.250000 & $0.01825(9)$ \\
C11 & $0.38953(3)$ & $0.10590(5)$ & $0.13863(3)$ & $0.02196(11)$ \\
$\mathrm{Cl} 2$ & $0.41898(3)$ & $0.32033(5)$ & $0.36739(4)$ & $0.02260(11)$ \\
$\mathrm{N} 1$ & $0.68501(11)$ & $0.01294(19)$ & $0.63521(12)$ & $0.0218(3)$ \\
$\mathrm{H} 1$ & 0.642734 & -0.054296 & 0.645210 & $0.026^{*}$ \\
$\mathrm{C} 2$ & $0.66434(12)$ & $0.1697(2)$ & $0.62543(13)$ & $0.0197(3)$ \\
$\mathrm{C} 3$ & $0.73176(13)$ & $0.2747(2)$ & $0.61076(14)$ & $0.0222(4)$ \\
$\mathrm{H} 3$ & 0.720458 & 0.386273 & 0.605979 & $0.027^{*}$ \\
$\mathrm{C} 4$ & $0.83354(14)$ & $0.0533(2)$ & $0.61276(16)$ & $0.0261(4)$ \\
$\mathrm{H} 4$ & 0.890816 & 0.012622 & 0.607122 & $0.031^{*}$ \\
$\mathrm{C} 5$ & $0.81548(14)$ & $0.2165(2)$ & $0.60311(15)$ & $0.0247(4)$ \\
$\mathrm{H} 5$ & 0.861102 & 0.288380 & 0.591178 & $0.030^{*}$ \\
$\mathrm{C} 6$ & $0.76632(14)$ & $-0.0468(2)$ & $0.63060(15)$ & $0.0254(4)$ \\
$\mathrm{H} 6$ & 0.777088 & -0.158092 & 0.639704 & $0.031^{*}$ \\
$\mathrm{C} 7$ & $0.57039(13)$ & $0.2174(2)$ & $0.62851(16)$ & $0.0259(4)$ \\
$\mathrm{H} 7 \mathrm{~A}$ & 0.536395 & 0.214343 & 0.556960 & $0.039^{*}$ \\
$\mathrm{H} 7 \mathrm{~B}$ & 0.569141 & 0.325976 & 0.656845 & $0.039^{*}$ \\
$\mathrm{H} 7 \mathrm{C}$ & 0.543936 & 0.143189 & 0.673767 & $0.039^{*}$ \\
& & & &
\end{tabular}

Atomic displacement parameters $\left(\AA^{2}\right)$

\begin{tabular}{lllllll}
\hline & $U^{11}$ & $U^{22}$ & $U^{33}$ & $U^{12}$ & $U^{13}$ & $U^{23}$ \\
\hline $\mathrm{Cu} 1$ & $0.02142(16)$ & $0.01465(15)$ & $0.01929(16)$ & 0.000 & $0.00510(12)$ & 0.000 \\
$\mathrm{C} 11$ & $0.0253(2)$ & $0.0174(2)$ & $0.0226(2)$ & $-0.00165(16)$ & $0.00223(17)$ & $-0.00185(15)$ \\
$\mathrm{Cl} 2$ & $0.0276(2)$ & $0.0172(2)$ & $0.0246(2)$ & $0.00035(16)$ & $0.00935(17)$ & $-0.00340(16)$ \\
$\mathrm{N} 1$ & $0.0268(8)$ & $0.0154(7)$ & $0.0235(7)$ & $-0.0023(6)$ & $0.0054(6)$ & $0.0011(6)$ \\
$\mathrm{C} 2$ & $0.0264(9)$ & $0.0172(8)$ & $0.0155(8)$ & $-0.0004(7)$ & $0.0033(7)$ & $0.0000(6)$ \\
$\mathrm{C} 3$ & $0.0311(10)$ & $0.0150(8)$ & $0.0203(8)$ & $-0.0030(7)$ & $0.0040(7)$ & $0.0004(6)$ \\
$\mathrm{C} 4$ & $0.0251(10)$ & $0.0266(10)$ & $0.0267(9)$ & $0.0016(8)$ & $0.0040(8)$ & $-0.0010(8)$ \\
$\mathrm{C} 5$ & $0.0281(10)$ & $0.0221(9)$ & $0.0244(9)$ & $-0.0069(7)$ & $0.0056(8)$ & $-0.0008(7)$ \\
$\mathrm{C} 6$ & $0.0326(10)$ & $0.0172(9)$ & $0.0265(10)$ & $0.0031(7)$ & $0.0048(8)$ & $0.0016(7)$ \\
$\mathrm{C} 7$ & $0.0263(10)$ & $0.0243(10)$ & $0.0276(10)$ & $0.0018(7)$ & $0.0061(8)$ & $0.0004(8)$ \\
\end{tabular}

Geometric parameters $\left(A,{ }^{\circ}\right)$

\begin{tabular}{llll}
\hline $\mathrm{Cu} 1-\mathrm{C} 11^{\mathrm{i}}$ & $2.2496(5)$ & $\mathrm{C} 3-\mathrm{C} 5$ & $1.383(3)$ \\
$\mathrm{Cu} 1-\mathrm{C} 11$ & $2.2496(5)$ & $\mathrm{C} 4-\mathrm{H} 4$ & 0.9500 \\
$\mathrm{Cu} 1-\mathrm{Cl} 2$ & $2.2492(4)$ & $\mathrm{C} 4-\mathrm{C} 5$ & $1.395(3)$ \\
$\mathrm{Cu} 1-\mathrm{Cl} 2^{\mathrm{i}}$ & $2.2492(4)$ & $\mathrm{C} 4-\mathrm{C} 6$ & $1.370(3)$
\end{tabular}




\begin{tabular}{|c|c|c|c|c|}
\hline $\mathrm{N} 1-\mathrm{H} 1$ & 0.8800 & $\mathrm{C} 5-\mathrm{H} 5$ & & 0.9500 \\
\hline $\mathrm{N} 1-\mathrm{C} 2$ & $1.350(2)$ & $\mathrm{C} 6-\mathrm{H} 6$ & & 0.9500 \\
\hline $\mathrm{N} 1-\mathrm{C} 6$ & $1.346(2)$ & $\mathrm{C} 7-\mathrm{H} 7 \mathrm{~A}$ & & 0.9800 \\
\hline $\mathrm{C} 2-\mathrm{C} 3$ & $1.387(3)$ & $\mathrm{C} 7-\mathrm{H} 7 \mathrm{~B}$ & & 0.9800 \\
\hline $\mathrm{C} 2-\mathrm{C} 7$ & $1.493(3)$ & $\mathrm{C} 7-\mathrm{H} 7 \mathrm{C}$ & & 0.9800 \\
\hline $\mathrm{C} 3-\mathrm{H} 3$ & 0.9500 & & & \\
\hline $\mathrm{Cl} 1-\mathrm{Cu} 1-\mathrm{Cl1}^{\mathrm{i}}$ & $128.54(3)$ & $\mathrm{C} 5-\mathrm{C} 4-\mathrm{H} 4$ & & 121.0 \\
\hline $\mathrm{Cl} 2^{\mathrm{i}}-\mathrm{Cu} 1-\mathrm{Cl} 1$ & $99.614(17)$ & $\mathrm{C} 6-\mathrm{C} 4-\mathrm{H} 4$ & & 121.0 \\
\hline $\mathrm{Cl} 2^{\mathrm{i}}-\mathrm{Cu} 1-\mathrm{C} 11^{\mathrm{i}}$ & $98.550(17)$ & $\mathrm{C} 6-\mathrm{C} 4-\mathrm{C} 5$ & & $118.10(19)$ \\
\hline $\mathrm{Cl} 2-\mathrm{Cu} 1-\mathrm{Cl} 1$ & $98.549(17)$ & $\mathrm{C} 3-\mathrm{C} 5-\mathrm{C} 4$ & & $120.61(18)$ \\
\hline $\mathrm{Cl} 2-\mathrm{Cu} 1-\mathrm{Cl}^{\mathrm{i}}$ & $99.616(17)$ & $\mathrm{C} 3-\mathrm{C} 5-\mathrm{H} 5$ & & 119.7 \\
\hline $\mathrm{Cl} 2 \mathrm{i}-\mathrm{Cu} 1-\mathrm{Cl} 2$ & $137.36(3)$ & $\mathrm{C} 4-\mathrm{C} 5-\mathrm{H} 5$ & & 119.7 \\
\hline $\mathrm{C} 2-\mathrm{N} 1-\mathrm{H} 1$ & 118.0 & $\mathrm{~N} 1-\mathrm{C} 6-\mathrm{C} 4$ & & $119.91(18)$ \\
\hline $\mathrm{C} 6-\mathrm{N} 1-\mathrm{H} 1$ & 118.0 & $\mathrm{~N} 1-\mathrm{C} 6-\mathrm{H} 6$ & & 120.0 \\
\hline $\mathrm{C} 6-\mathrm{N} 1-\mathrm{C} 2$ & $124.00(17)$ & $\mathrm{C} 4-\mathrm{C} 6-\mathrm{H} 6$ & & 120.0 \\
\hline $\mathrm{N} 1-\mathrm{C} 2-\mathrm{C} 3$ & $117.46(17)$ & $\mathrm{C} 2-\mathrm{C} 7-\mathrm{H} 7 \mathrm{~A}$ & & 109.5 \\
\hline $\mathrm{N} 1-\mathrm{C} 2-\mathrm{C} 7$ & $117.88(17)$ & $\mathrm{C} 2-\mathrm{C} 7-\mathrm{H} 7 \mathrm{~B}$ & & 109.5 \\
\hline $\mathrm{C} 3-\mathrm{C} 2-\mathrm{C} 7$ & $124.64(17)$ & $\mathrm{C} 2-\mathrm{C} 7-\mathrm{H} 7 \mathrm{C}$ & & 109.5 \\
\hline $\mathrm{C} 2-\mathrm{C} 3-\mathrm{H} 3$ & 120.1 & $\mathrm{H} 7 \mathrm{~A}-\mathrm{C} 7-\mathrm{H} 7 \mathrm{~B}$ & & 109.5 \\
\hline $\mathrm{C} 5-\mathrm{C} 3-\mathrm{C} 2$ & $119.88(17)$ & $\mathrm{H} 7 \mathrm{~A}-\mathrm{C} 7-\mathrm{H} 7 \mathrm{C}$ & & 109.5 \\
\hline $\mathrm{C} 5-\mathrm{C} 3-\mathrm{H} 3$ & 120.1 & $\mathrm{H} 7 \mathrm{~B}-\mathrm{C} 7-\mathrm{H} 7 \mathrm{C}$ & & 109.5 \\
\hline $\mathrm{N} 1-\mathrm{C} 2-\mathrm{C} 3-\mathrm{C} 5$ & $2.2(3)$ & $\mathrm{C} 6-\mathrm{N} 1-\mathrm{C} 2-\mathrm{C} 3$ & & $-0.6(3)$ \\
\hline $\mathrm{C} 2-\mathrm{N} 1-\mathrm{C} 6-\mathrm{C} 4$ & $-1.5(3)$ & $\mathrm{C} 6-\mathrm{N} 1-\mathrm{C} 2-\mathrm{C} 7$ & & $177.92(17)$ \\
\hline $\mathrm{C} 2-\mathrm{C} 3-\mathrm{C} 5-\mathrm{C} 4$ & $-1.7(3)$ & $\mathrm{C} 6-\mathrm{C} 4-\mathrm{C} 5-\mathrm{C} 3$ & & $-0.4(3)$ \\
\hline $\mathrm{C} 5-\mathrm{C} 4-\mathrm{C} 6-\mathrm{N} 1$ & $2.0(3)$ & $\mathrm{C} 7-\mathrm{C} 2-\mathrm{C} 3-\mathrm{C} 5$ & & $-176.27(18)$ \\
\hline \multicolumn{5}{|c|}{ Symmetry code: (i) $-x+1, y,-z+1 / 2$. } \\
\hline \multicolumn{5}{|c|}{ Hydrogen-bond geometry $\left(\AA,{ }^{\circ}\right)$} \\
\hline$D-\mathrm{H} \cdots A$ & $D-\mathrm{H}$ & $\mathrm{H} \cdots A$ & $D \cdots A$ & $D-\mathrm{H} \cdots A$ \\
\hline $\mathrm{N} 1-\mathrm{H} 1 \cdots \mathrm{Cl} 1^{\mathrm{ii}}$ & 0.88 & 2.93 & $3.4297(16)$ & 118 \\
\hline $\mathrm{N} 1-\mathrm{H} 1 \cdots \mathrm{Cl} 2^{\mathrm{ii}}$ & 0.88 & 2.41 & $3.2050(16)$ & 150 \\
\hline $\mathrm{C} 6-\mathrm{H} 6 \cdots \mathrm{C} 11^{\mathrm{iii}}$ & 0.95 & 2.62 & $3.453(2)$ & 147 \\
\hline $\mathrm{C} 7-\mathrm{H} 7 A \cdots \mathrm{Cl} 2$ & 0.98 & 2.92 & $3.850(2)$ & 159 \\
\hline $\mathrm{C} 7-\mathrm{H} 7 B \cdots \mathrm{Cl} 2^{\mathrm{iv}}$ & 0.98 & 2.98 & $3.872(2)$ & 151 \\
\hline
\end{tabular}

Symmetry codes: (ii) $-x+1,-y,-z+1$; (iii) $x+1 / 2, y-1 / 2, z+1 / 2$; (iv) $-x+1,-y+1,-z+1$. 